\title{
Antibiotic adjuvants to rescue Pseudomonas aeruginosa from tetracycline antibiotics
}

\section{resistance}

Azza Troudi $^{1 ¥}$, Hana Douafer ${ }^{1 ¥}$, Jean-Michel Bolla ${ }^{1}$, Naouel Klibi ${ }^{2}$ and Jean Michel Brunel ${ }^{1 *}$

1 Aix Marseille Univ, INSERM, SSA, MCT, 13385 Marseille, France. E-mail : bruneljm@yahoo.fr

${ }^{2}$ Laboratory of Microorganisms and Active Biomolecules, Department of Biology, Faculty of Sciences of Tunis, University of Tunis El Manar, Tunis, Tunisia.

$¥$ A. T. and H. D. contributed equally to this work

\begin{abstract}
An attractive antibiotic-adjuvant strategy consisting in the design and synthesis of polyaminoisoprenyl molecules able to restore antibiotic activity of tetracycline antibiotics against resistant Pseudomonas aeruginosa bacterial strains has been developed. These chemosensitizers are readily prepared from geraniol and farnesol in an efficient two steps synthesis with good to moderate yields varying from 38 to $64 \%$ and leading to a significant decrease of antibiotic resistance. Thus, the influence of the nature of the tetracycline antibiotic used as well as the structure of the polyaminoisoprenyl derivatives involved on the outcome of the antibiotic-adjuvant combination against $P$. aeruginosa resistance to tetracyclines were investigated. Additionally, our data suggested that their mechanism of action is closely associated with the increase of the outer-membrane permeability.
\end{abstract}

\section{Keywords}

Polyaminoisoprenyl derivatives - Antibiotic adjuvants - Polyamines - Pseudomonas aeruginosa - Tetracycline antibiotic

\section{Introduction}

Since the discovery of antibiotics in 1928 by Alexander Fleming, their massive and/or inappropriate use during the last century has led to the emergence of antimicrobial resistance. ${ }^{[1-3]}$ During the last decades, we witnessed the selection of resistant bacteria whose genome has been modified either by mutation and/or acquisition of genetic material, favoring 
their development at the expense of sensitive ones. This phenomenon of antimicrobial resistance constantly expanding compromises the existing antibiotic arsenal and leads more and more clinicians to therapeutic impasses especially concerning infections by Gramnegative bacteria which constitutes one of the major current threats to public health. ${ }^{[4-5]}$ In this context, Pseudomonas aeruginosa which is an opportunistic pathogen that rarely causes disease in healthy people but is responsible for serious infections in patients with cystic fibrosis and severe burns as well as immunocompromised patients lead to a high mortality rate in this population. ${ }^{[6-10]}$ Thus, there is an urgent need for the development of therapeutic agents that can modify the bacterial outer membrane integrity and/or its permeability since this latter greatly limits the diffusion of numerous hydrophilic antibiotic molecules. Without an innovative strategy to combat multi-drug resistant (MDR) pathogens, many fields of medicine will be severely affected. In this context, new solutions are required to enhance the antibiotic efficiency and/or reduce the mechanism of resistance. Thus, the concept of antibiotic adjuvants, able to re-empower the antibiotic agent activity to become efficient against the resistant strain of interest could constitute a valuable strategy. For that an ideal adjuvant must meet the following requirements: (1) be specific for the bacterial target and ideally free from any pharmacological activity on eukaryotic cells, (2) be able to reach a therapeutically effective concentration in serum and reach its target in vivo and (3) be devoid of antibacterial activity to lower the possibility of the selection of resistance mechanisms. ${ }^{[11]}$ Recently, we reported the ability of polyamino-isoprenyl compound $\mathbf{3}$ to potentiate the florfenicol activity in theBordetella bronchiseptica porcine pulmonary infection treatment. ${ }^{[12]}$ In the context of our studies, we will describe herein that a reasoned adjuvant design based on substituted polyaminoisoprenyl molecules made of a terpene core and bearing various polyamine groups permits one to perform polypharmacy on $P$. aeruginosa bacteria by providing greater internal access to inactive tetracycline antibiotics. ${ }^{[13]}$

\section{Results and discussion}

\subsection{Polyaminoisoprenyl derivatives synthesis}

For our preliminary optimization studies, we chose to focus on the synthesis of derivative $\mathbf{3}$ under various experimental conditions according to the following synthetic pathway (Scheme 1). It clearly appears that performing the reaction in THF permits to obtain the expected compound in $64 \%$ isolated yield whereas lower yields were encountered in $\mathrm{CH}_{2} \mathrm{Cl}_{2}$, toluene or DMF ranging from 42,51 and $22 \%$ respectively. This strategy involving a direct nucleophilic substitution of the considered polyamine on farnesyl chloride $\mathbf{1}$ and geranyl chloride $\mathbf{2}$ 
derivative was then successfully applied to the synthesis of derivatives 4-9 (Scheme1). In all cases, the expected adducts were obtained in moderate to good not optimized yields varying from 38 to $61 \%$ depending on the nature of the considered polyamine.
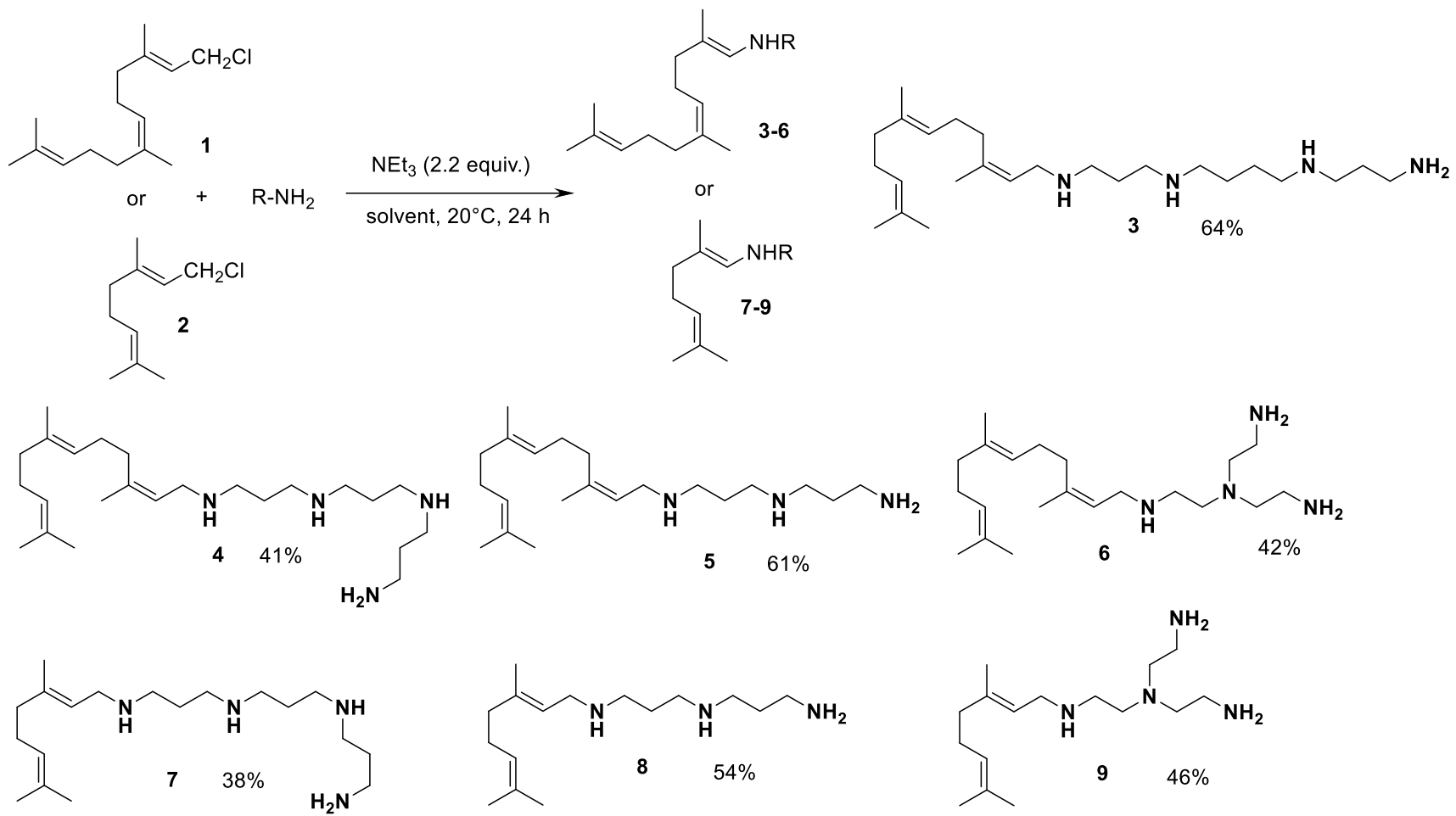

Scheme 1. Synthesis of polyaminofarnesyl and polyaminogeranyl derivatives 3-9

\subsection{Characteristics of the bacterial strains and MIC determination}

The seven molecules 3-9 were evaluated for their MICs against $P$. aeruginosa PA01 bacterial strain in order to allow us to determine the amount of product that could be added to each strain without producing a direct antibacterial activity. As summarized in Table 1, all the compounds demonstrated MICs varying from 100 to $400 \mu \mathrm{M}$ except for compound 3 which lead to a MIC of $25 \mu \mathrm{M}$. It is also noteworthy that a MIC of $144 \mu \mathrm{M}(64 \mu \mathrm{g} / \mathrm{mL})$ was encountered for doxycycline under the same experimental conditions. 


\begin{tabular}{ccc}
\hline Entry & Compound & MIC $(\boldsymbol{\mu M})$ \\
\hline 1 & $\mathbf{3}$ & 25 \\
2 & $\mathbf{4}$ & 100 \\
3 & $\mathbf{5}$ & 100 \\
4 & $\mathbf{6}$ & 100 \\
5 & $\mathbf{7}$ & $>400$ \\
6 & $\mathbf{8}$ & $>400$ \\
7 & $\mathbf{9}$ & $>400$ \\
& & 144 \\
8 & Doxycycline & $(64 \mu \mathrm{g} / \mathrm{mL})$
\end{tabular}

Table 1. MICs of doxycycline and of the derivatives 3-9 against P.aeruginosa PA01

The compounds were then all assayed for their ability to potentiate doxycycline activity against $P$. aeruginosa PA01 at 3 different fixed concentrations $(2.5,5,10 \mu \mathrm{M})$.

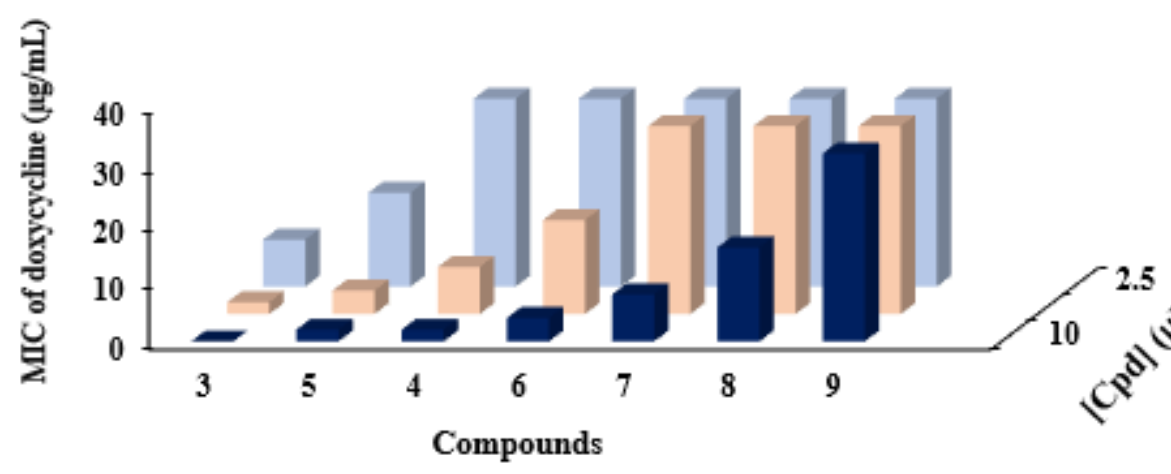

\begin{tabular}{cccccccc}
\hline Compounds & $\mathbf{3}$ & $\mathbf{4}$ & $\mathbf{5}$ & $\mathbf{6}$ & $\mathbf{7}$ & $\mathbf{8}$ & $\mathbf{9}$ \\
\hline Gain factor $(\mathbf{1 0} \boldsymbol{\mu M})$ & $\mathbf{1 2 8}$ & 32 & 32 & 16 & 4 & 8 & 2 \\
Gain factor $(2.5 \boldsymbol{\mu M})$ & 8 & 2 & 4 & 2 & 2 & 2 & 2 \\
\hline
\end{tabular}

Figure 1. Dose-dependent effect of the different compounds in combination with doxycycline against $P$. aeruginosa PA01 ( 3 coloured bars for $2.5,5$ and, $10 \mu \mathrm{M}$ )

The results clearly indicate a dose-dependent potentiation of the doxycycline activity in presence of the different derivatives tested, except for compound $\mathbf{9}$ showing no efficiency in the concentration range tested. In addition, we identified two groups of compounds, one group constituted by the geranyl derivatives 7-9 that had a weak chemosensitizing activity and a second one consisting in farnesyl compounds 3-6 that exhibited a high efficiency for increasing doxycycline susceptibility. Amongst them, the best compound restoring the efficacy of doxycycline was identified as the polyaminoisoprenyl derivative $\mathbf{3}$ which induced 
a decrease in the MIC of doxycycline from $64 \mu \mathrm{g} / \mathrm{mL}$ to $0.5 \mu \mathrm{g} / \mathrm{mL}$ with a gain factor of 128 fold when used at a $10 \mu \mathrm{M}$ concentration (the gain factor corresponding to the ratio of the MIC of the antibiotic tested alone to the MIC of the antibiotic obtained in the presence of the considered adjuvant). This concentration could be considered high compared to a compound used as an adjuvant. Therefore, we determined the concentration of each compound necessary to restore the MIC of doxycycline to the sensitivity threshold $(2 \mu \mathrm{g} / \mathrm{mL})$ against $P$. aeruginosa PA01 (Table 2).

\begin{tabular}{ccc}
\hline Entry & Compound & $\begin{array}{c}\text { Concentration } \\
(\boldsymbol{\mu M})\end{array}$ \\
\hline 1 & $\mathbf{3}$ & 1.25 \\
2 & $\mathbf{4}$ & 6.25 \\
3 & $\mathbf{5}$ & 6.25 \\
4 & $\mathbf{6}$ & 12.5 \\
5 & $\mathbf{7}$ & 25 \\
6 & $\mathbf{8}$ & 50 \\
7 & $\mathbf{9}$ & 100
\end{tabular}

Table 2. Concentrations of the different compounds used to restore doxycycline activity at a 2 $\mu \mathrm{g} / \mathrm{mL}$ concentration against $P$.aeruginosa PA01

All these data confirmed that compound $\mathbf{3}$ possesses the best adjuvant properties among the tested compounds since a concentration of only $1.25 \mu \mathrm{M}(1.30 \mu \mathrm{g} / \mathrm{mL})$ permits to decrease the MIC of doxycycline against $P$. aeruginosa at the sensitivity level of $2 \mu \mathrm{g} / \mathrm{mL}$. The other compounds should be used at slightly higher concentrations in the case of the farnesyl group derivatives $(6.25 \mu \mathrm{M}$ for 4 and 5$)$ or moderately high for $6(12.5 \mu \mathrm{M})$ whereas the geranyl group compounds should be used at very high concentrations as for $\mathbf{9}$ with a concentration up to $100 \mu \mathrm{M}$.

As an extension of this study, we have investigated the use of various tetracycline antibiotics and determine the different antibiotic-adjuvant combination efficiency against PA01 (Figure 2). 


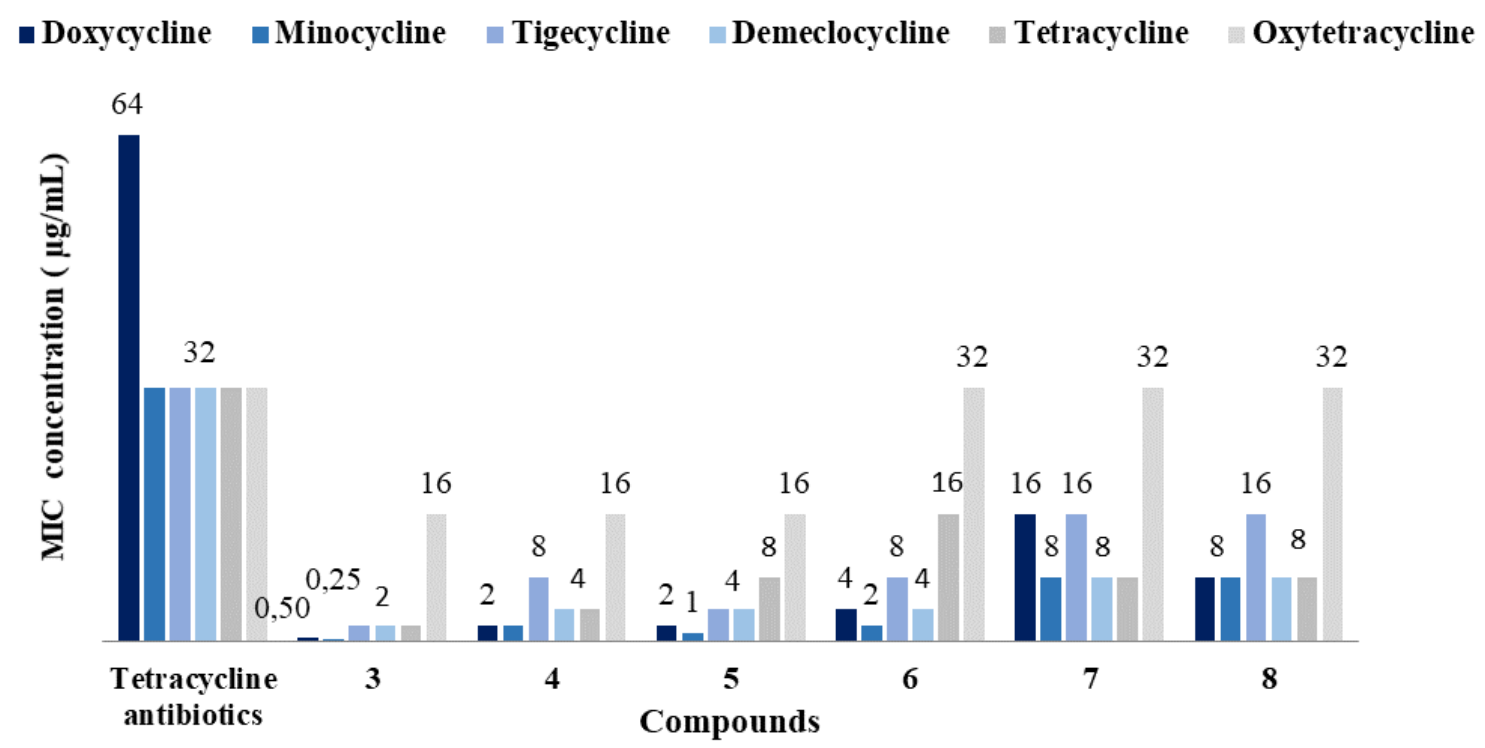

Figure 2. Restoration of tetracycline activity against P.aeruginosa PA01 in the presence of a $10 \mu \mathrm{M}$ concentration of derivatives 3-8

Thus, their antibacterial activity in the presence or absence of polyamino-isoprenyl adjuvants at a $10 \mu \mathrm{M}$ concentration against $P$. aeruginosa PA01 was determined. Among all the antibiotics tested, minocycline and doxycycline in combinations with different adjuvants led to the best results. On the other hand, we proved that numerous of these polyaminoisoprenyl derivatives possess antibiotic enhancing properties of $P$. aeruginosa reference strain but that polyamino-farnesyl compounds demonstrate better efficiency compared to polyamino-geranyl derivatives. Altogether these results demonstrated that derivatives $\mathbf{3}$ and $\mathbf{5}$ represent good candidates for circumventing $P$. aeruginosa tetracycline antibiotics resistance (Figure $3 \mathrm{a}$ ). 

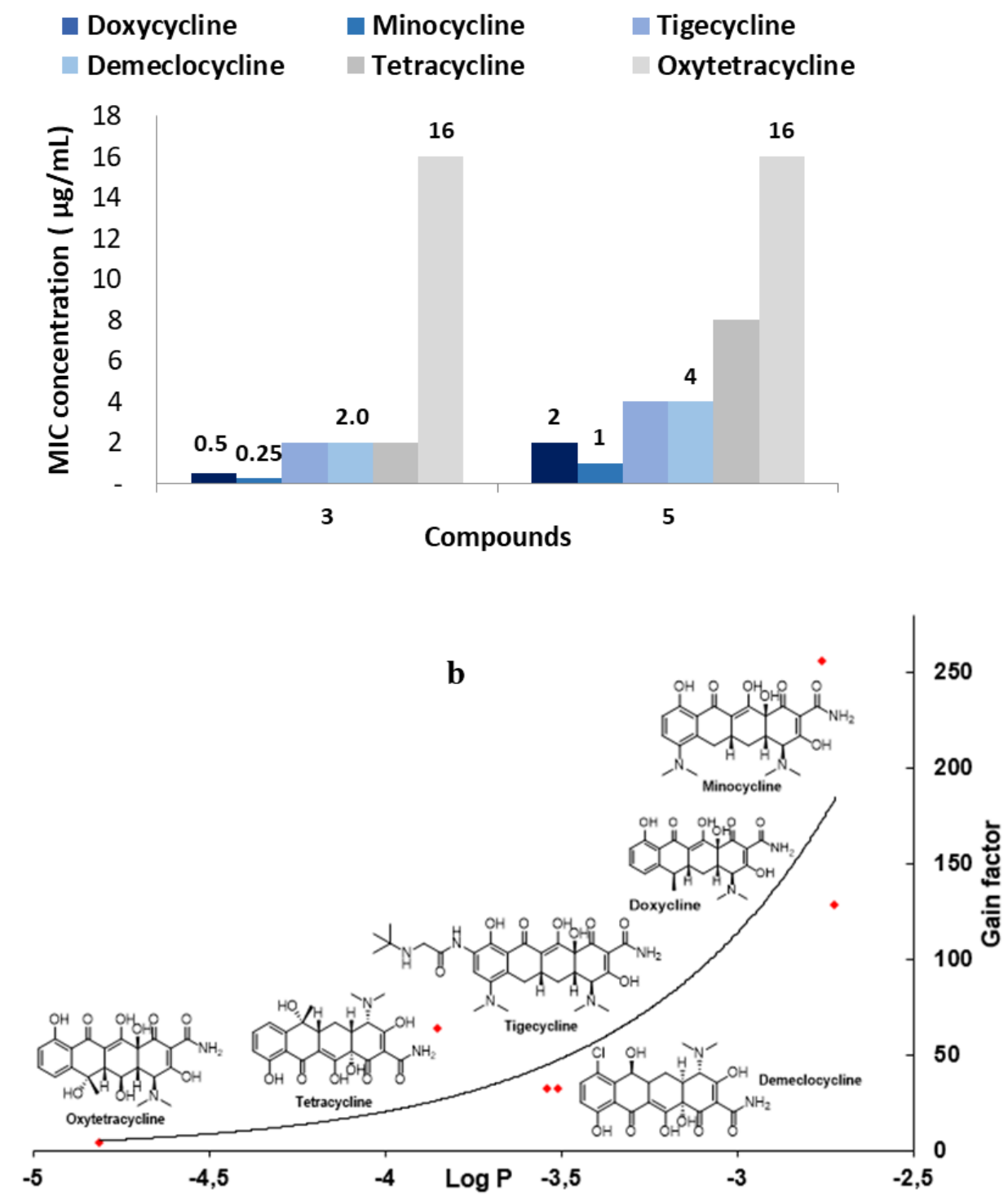

Figure 3. a) Restoration of tetracycline antibiotics activity against PA01 in the presence of derivatives $\mathbf{3}$ and 5 (10 $\mu \mathrm{M}$ concentration). b) Correlation of the gain factor observed depending on the $\log \mathrm{P}$ value of the considered tetracycline antibiotic.

Thus, the results from these latter cases also suggest that the steric hindrance generated, and hydrophobic nature of the farnesyl group constitute the most important factors to destabilize the integrity of the outer membrane of the bacteria. On the other hand, taking into consideration for each tetracycline antibiotic tested the $\log P$ parameter which reflects the true behavior and bioavailability of an ionizable compound in a solution, a significant correlation with the gain factor activity was observed (Figure 3b). Indeed, those presenting the highest LogP values (e.g., the most hydrophobic derivatives minocycline and doxycycline), and therefore the better affinity with the destabilized outer membrane to penetrate the bacteria, also displayed higher gain factor activity. 
In order to study their mechanism of action, these compounds were evaluated for their effect on the integrity of the outer membrane of P. aeruginosa PA01 cells. The method used is based on the study of the chromogenic $\beta$-lactam nitrocefin hydrolysis kinetic of which hydrolysis by periplasmic beta-lactamases cause a change in color from yellow to red permitting to relate it to the integrity of the outer membrane. ${ }^{[14-16]}$ Thus, the curves of nitrocefin hydrolysis kinetic in the presence of derivatives $\mathbf{3}$ and $\mathbf{8}$ as well as two positive controls polymyxin B PMB and polymyxin B nona (PMBn) known for their permeabilizing effect of the external bacterial membrane are reported in Figure 4. At the same concentration of $128 \mu \mathrm{M}$, the results showed a strong and rapid disruption of the bacterial membrane by the farnesyl derivative 3 as for positive control PMB. This effect was revealed by a rapid increase in the nitrocefin hydrolysis rate. However, geranyl derivative $\mathbf{8}$ acts slowly on the bacterial membrane giving a hydrolysis kinetic profile as the one obtained with PMBn. It is noteworthy that these results are in good correlation with those obtained in our previous antibioticadjuvant tests (Table 2).

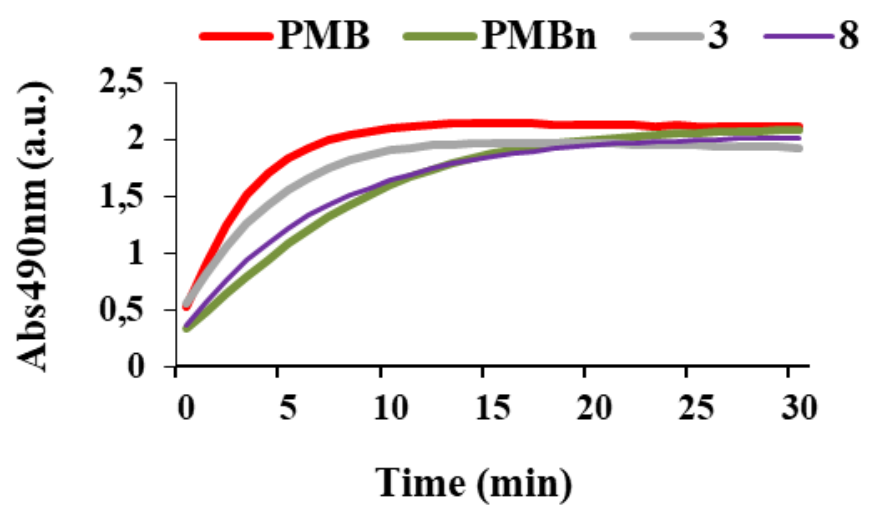

Figure 4. Comparison of the nitrocefin hydrolysis kinetics in the presence of PMB, PMBn, 3 and 8 used at a $128 \mu \mathrm{M}$ concentration.

The resemblance percentages (Figure S3)of the effect on the membrane integrity caused by each compound $(64 \mu \mathrm{M})$ to that of $\mathrm{PMB}$ or $\mathrm{PMBn}$ are summarized in Figure $5 \mathrm{a}$ and $5 \mathrm{~b}$, respectively. 


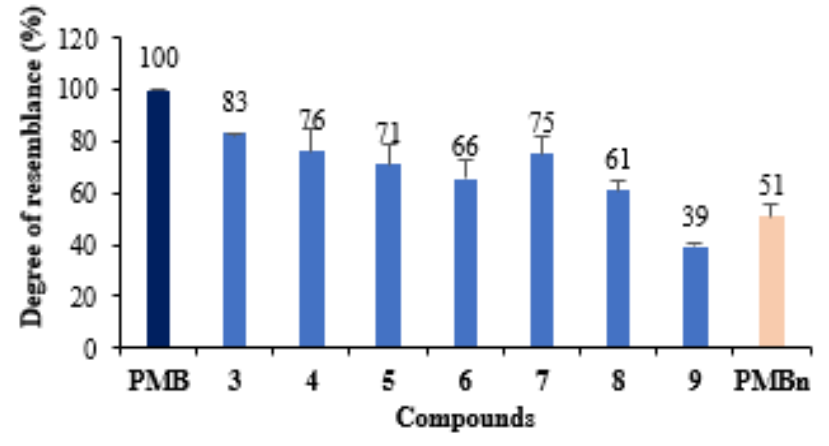

b

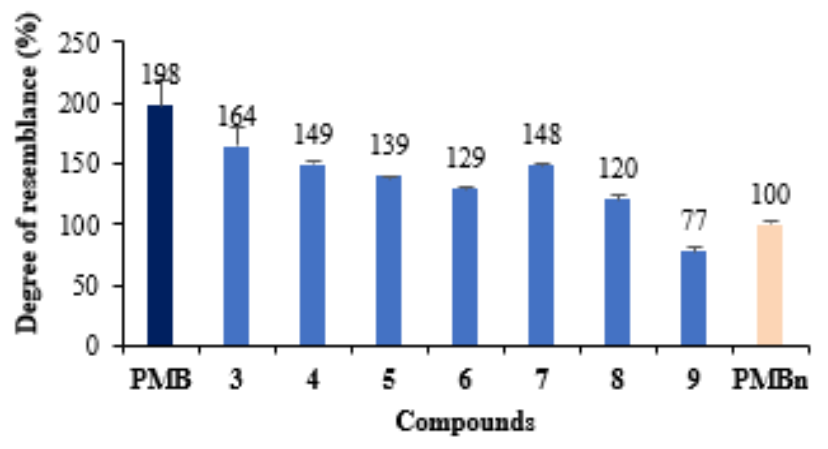

Figure 5. Comparison of membrane permeation effects of the different compounds with those of PMB (a) and PMBn (b) (all used at a $64 \mu \mathrm{M}$ concentration)

It is noteworthy that lower concentrations $(32,16 \mu \mathrm{M})$ were tested for each compound and lead to a similar response demonstrating that this effect is dose dependent (data not shown). Additionally, by performing experiment against $P$. aeruginosa PA403 efflux pump deficient mutant (mex $\mathrm{AB}, m e x \mathrm{CD}, m e x \mathrm{EF}, m e x \mathrm{XY}, m e x \mathrm{JK})$ and $P$. aeruginosa PA01 OprM deficient ${ }^{[17-19]}$ some assumptions can be highlighted (Table 3).

\begin{tabular}{cccc}
\hline Strains & $\begin{array}{c}\text { MIC of doxycycline } \\
(\mu \mathrm{g} / \mathrm{mL})\end{array}$ & MIC of $\mathbf{3}(\mu \mathrm{M})$ & $\begin{array}{c}\text { MIC of doxycycline in the } \\
\text { presence of } \mathbf{3}(2.5 \mu \mathrm{M})\end{array}$ \\
\hline PA01 & 32 & 25 & 2 \\
PA01 $(\Delta$ oprM) & 32 & 25 & 1 \\
PA403 & 1 & 6.25 & 0.0156 \\
\hline
\end{tabular}

Table 3. Comparison of doxycycline activity in the presence or absence of compound $\mathbf{3}$ against $P$. aeruginosa mutant strains

Thus, 3 can be considered as a potent substrate of CD, EF and/or JK efflux pumps since a weak variation of its MIC is noticed against both PA01, PA403 and PA01 ( $\Delta o p r \mathrm{M})$. Moreover, we can notice the low influence of $o p r \mathrm{M}$ deletion on the MICs obtained for doxycycline and compound $\mathbf{3}$ suggesting that $\mathrm{CD}$ and EF pumps can expel doxycycline outside from the bacteria. Finally, by considering PA403 strain the intracellular concentration of doxycycline is increased in the presence of derivative 3 since a MIC of doxycycline of $0.0078 \mu \mathrm{g} / \mathrm{mL}$ is encountered corresponding to 128 times fold that which is observed against PA01.

All our data suggest that these polyaminoisoprenyl derivatives disrupt the outer membrane integrity of Gram-negative bacteria in a dose-dependent manner. Nevertheless, the farnesyl group compounds (3-6) confers a greater permeabilizing effect to the compounds that what obtained in the presence of the geranyl group derivatives (7-9). 
Finally, a time-kill assay against $P$. aeruginosa PA01 involving different 3-doxycycline combination concentrations has been performed (Figure 6).

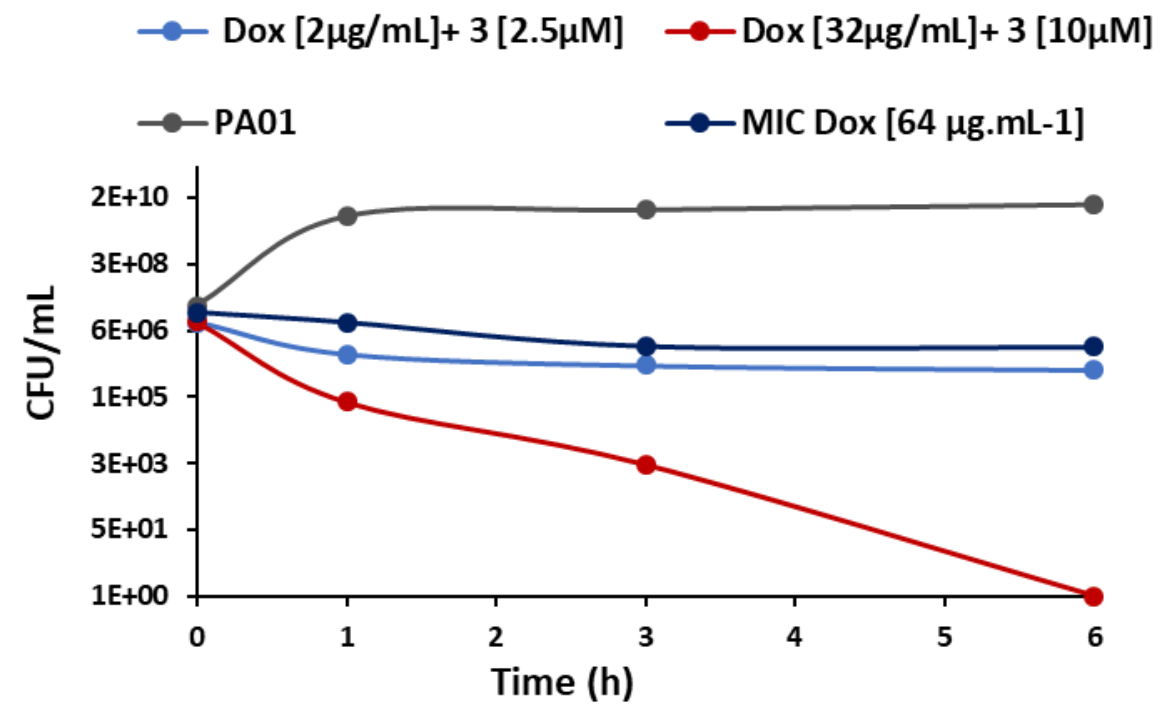

Figure 6.

No significant differences were measured between the time kill profile of doxycycline at its own MIC $(64 \mu \mathrm{g} / \mathrm{mL})$ and the combination of doxycycline with compound 3 used at concentrations of $8 \mu \mathrm{g} / \mathrm{mL}$ and $2.5 \mu \mathrm{M}$, respectively. They therefore have a bacteriostatic effect since the Colony Forming Unit number has remained the same over time as the original inoculum. Nevertheless, exposure of PA01 to a combination of doxycycline-3 used at concentrations of $32 \mu \mathrm{g} / \mathrm{mL}$ and $10 \mu \mathrm{M}(10 \mu \mathrm{g} / \mathrm{mL})$, respectively, was able to induce a 2 $\log _{10}$ reduction from the initial inoculum after $3 \mathrm{~h}$ incubation whereas a complete killing was achieved within 6 hours. Thus, this result indicates a bactericidal effect of the doxycycline-3 combination each used at $32 \mu \mathrm{g} / \mathrm{mL}$ and $10 \mu \mathrm{M}$, respectively.

\section{Conclusion}

An original chemical strategy has been developed affording new polyaminoisoprenyl compounds in moderate to good yields. Amongst all the synthetized farnesyl derivatives group 3-6 exhibited a strong effect on the level of tetracycline antibiotics susceptibility for resistant $P$. aeruginosa bacterial strains. This activity was correlated to the level of hydrophobicity of the antibiotics as well as the ability of the polyaminoisoprenyl derivatives to alter bacterial outer membrane integrity. Studies are now underway to determine if this restoration of antibiotic susceptibility occurs also by a direct interaction of the molecule with the efflux pump or by another mechanism. 


\section{References}

1. Livermore, D. M. The need for new antibiotics. Clin. Microbiol. Infect. 2004, 10, 1-9

2. Aslam, B.; Wang, W.; Arshad, M. I.; Khurshid, M.; Muzammil, S.; Rasool, M. H.; Nisar, M. A.; Alvi, R. F.; Aslam, M. A.; Qamar, M. U.; Salamat, M. K. F.; Baloch, Z. Antibiotic resistance: a rundown of a global crisis. Infect Drug Resist. 2018, 11, 1645-1658.

3. Payne, D. J. Microbiology: desperately seeking new antibiotics. Science 2006, 321, 16441645 .

4. Vivas, R.; Barbosa, A. A. T.; Dolabela, S. S.; Jain, S. Multidrug-resistant bacteria and alternative methods to control them: An overview. Microb Drug Resist. 2019, 25, 890908.

5. Peterson, E.; Kaur, P. Antibiotic resistance mechanisms in bacteria: Relationships between resistance determinants of antibiotic producers, environmental bacteria, and clinical pathogen. Front Microbiol. 2018, 9, 2928.

6. Pang, Z.; Raudonis, R.; Glick, B. R.; Lin T. J.; Cheng, Z. Antibiotic resistance in Pseudomonas aeruginosa: mechanisms and alternative therapeutic strategies. Biotechnol Adv. 2019, 37, 177-192.

7. Sharma, G.; Rao, S.; Bansal, A.; Dang, S.; Gupta, S.; Gabrani, R. Pseudomonas aeruginosa biofilm: potential therapeutic targets. Biologicals 2014, 42, 1-7.

8. Manfredi, R. ; Nanetti, A.; Ferri, M., Chiodo, F. Pseudomonas spp. complications in patients with HIV disease: an eight-year clinical and microbiological survey. Eur $J$ Epidemiol. 2000, 16, 111-118.

9. Bouza, E.; Burillo, A.; Munoz, P. Catheter-related infections: diagnosis and intravascular treatment. Clin Microbiol Infect. 2002, 8, 265-274.

10. Valderrey, A. D.; Pozuelo, M. J.; Jimenez, P. A.; Macia, M. D.; Oliver, A.; Rotger, R. chronic colonization by Pseudomonas aeruginosa of patients with obstructive lung diseases: cystic fibrosis, bronchiectasis, and chronic obstructive pulmonary disease. Diagn Microbiol Infect Dis. 2010, 68, 20-27.

11. Douafer, H.; Andrieu, V.; Phanstiel, O.; Brunel, J. M. Antibiotic adjuvants : Make antibiotics great again ! J Med Chem. 2019, 62, 8665-8681.

12. Borselli, D.; Brunel, J. M.; Gorgé, O.; Bolla, J. M. Polyamino-isoprenyl derivatives as antibiotic adjuvants and motility inhibitors for Bordetella bronchiseptica porcine pulmonary infection treatment. Front Microbiol. 2019, 10, 1771. 
13. Borselli, D.; Lieutaud, A.; Thefenne, H., Garnotel, E.; Pagès, J. M.; Brunel, J. M.; Bolla, J. M. Polyamino-isoprenic derivatives block intrinsic resistance of $\mathrm{P}$. aeruginosa to doxycyline and chloramphenicol in vitro. Plos One. 2016, 11, e154490.

14. Lomovskaya, O.; Warren, M. S.; Lee, A.; Galazzo, J.; Fronko, R.; Lee, M.; Blais, J.; Cho, D.; Chamberland, S.; Renau, T.; Leger, R.; Hecker, S.; Watkins, W.; Hoshino, K.; Ishida, H.; Lee, V. J. Identification and characterization of inhibitors of multidrug resistance efflux pumps in Pseudomonas aeruginosa: novel agents for combination therapy. Antimicrob Agents Chemother. 2001, 45, 105-116.

15. Matsumoto, Y.; Hayama, K.; Sakakihara, S.; Nishino, K.; Noji, H.; Yamahuchi, A. Evaluation of multidrug efflux pumps inhibitors by a new method using microfluidic channels. Plos One 2011, 6, e18547.

16. Lome, V.; Brunel, J. M.; Pages, J. M.; Bolla, J. M. Multiparametric profiling for identification of chemosensitizers against Gram-negative bacteria. Front Microbiol. 2018, 9, 204-213.

17. Morita, Y.; Kimura, N.; Mima, N.; Mizushima, T.; Tsuchiya, T. Roles of MExXY- and MexAB multidrug efflux pumps in intrinsic multidrug resistance of Pseudomonas aeruginosa PA01. J Gen Appl Microbiol. 2001, 47, 27-32.

18. Li, X. Z.; Nikaido, H. Efflux-mediated drug resistance in bacteria: an update. Drugs 2009, 69, 1555-1623.

19. Lister, P. D.; Wolter, D. J.; Hanson, N. D. Antibacterial-resistant Pseudomonas aeruginosa: clinical impact and complex regulation of chromosomally encoded resistance mechanisms. Clin Microbiol Rev. 2009, 22, 582-610.

\section{Acknowledgments}

We thank Pr F. Van bambeke for providing us PA403 and PA01( 


\section{Graphical abstract}

Antibiotic adjuvants to rescue Pseudomonas aeruginosa from tetracycline antibiotics resistance. Azza Troudi, Hana Douafer, Jean-Michel Bolla, Naouel Klibi and Jean Michel Brunel

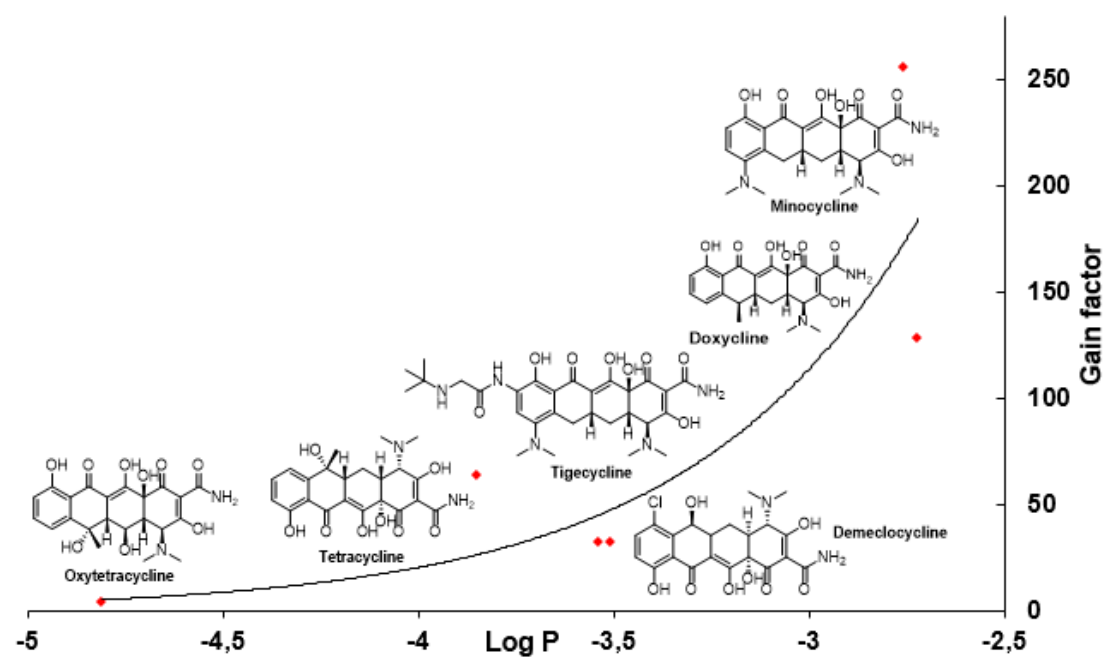

